\title{
Constraints on the local interstellar magnetic field from non-thermal emission of SN1006
}

\author{
F. Bocchino ${ }^{1}$, S. Orlando ${ }^{1}$, M. Miceli ${ }^{2}$, and O. Petruk ${ }^{3}$ \\ 1 INAF - Osservatorio Astronomico di Palermo, Piazza del Parlamento 1, 90134 Palermo, Italy \\ e-mail: bocchino@astropa.inaf.it \\ 2 Dipartimento di Scienze Fisiche ed Astronomiche, Sezione di Astronomia, Università di Palermo, Piazza del Parlamento 1, \\ 90134 Palermo, Italy \\ ${ }^{3}$ Institute for Applied Problems in Mechanics and Mathematics, Naukova St. 3-b, 79060 Lviv, Ukraine
}

Received 16 December 2010 / Accepted 29 April 2011

\begin{abstract}
Context. The synchrotron radio morphology of bilateral supernova remnants depends on the mechanisms of particle acceleration and on the viewing geometry. However, unlike X-ray and $\gamma$-ray morphologies, the radio emission does not depend on the cut-off region of the parent electron population, making it a simpler and more straightforward tool to investigate the physics of cosmic ray production in supernova remnants (SNRs).

Aims. We will use the radio morphology to derive tight constraints on the direction of the local magnetic field and its gradient, and on the obliquity dependence of the electron injection efficiency.

Methods. We perform a set of 3D MHD simulations describing the expansion of a spherical SNR through a magnetized medium with a non-uniform magnetic field. From the simulations, we derive non-thermal radio maps and compare them with observations of the SN1006 remnant.

Results. We find that the radio morphology of SN1006 at $1 \mathrm{GHz}$ is best-fitted by a model with quasi-parallel injection efficiency, a magnetic field aspect angle of $38^{\circ} \pm 4^{\circ}$ with the line of sight, and a gradient of the field strength toward the galactic plane, higher then the expected variations of the large scale field of the Galaxy.

Conclusions. We conclude that the radio limbs of SN1006 are polar caps that do not lie in the plane of sky. The study of the synchrotron radio emission of SNRs is of crucial importance to derive information on the galactic magnetic field in the vicinity of the remnants, and to gather more hints on the actual injection efficiency scenario.
\end{abstract}

Key words. acceleration of particles - shock waves - ISM: supernova remnants

\section{Introduction}

The radio morphology of supernova remnants (SNRs) may be very informative on the conditions of the magnetized environments in which the blast-wave expands and, more in particular, on the acceleration processes that occur at the shock front and that give rise to the energetic electrons ultimately responsible for the synchrotron emission in the radio and X-ray band. In particular, the radio emission is insensitive to accelerationrate issues, unlike the X-ray emission which depends on both the efficiency of electron injection into the acceleration process (i.e. the fraction of particles injected from the thermal gas, as in e.g. Blasi et al. 2005) and on the rapidity of acceleration to high energies. Indeed, the time required to accelerate an electron-emitting synchrotron emission at a frequency $v$ is about $3 \times 10^{-3} \eta(v / \mathrm{GHz})^{0.5}(B / 100 \mu \mathrm{G})^{-1.5}\left(V_{\mathrm{s}} / 3000 \mathrm{~km} \mathrm{~s}^{-1}\right)^{-2} \mathrm{yr}$ (Uchiyama et al. 2007), where $B$ is the magnetic field, $V_{\mathrm{s}}$ is the shock speed, $\eta$ is the ratio between the mean free path of the particles along the magnetic field line to the gyroradius, and the parameters are normalized to typical young SNR values. For the archetypical remnant SN1006, we have $B \sim 100 \mu \mathrm{G}$ (Völk et al. 2005; Ballet 2006; Morlino et al. 2010; Petruk et al. 2011), $V_{\mathrm{s}} \sim 4000 \mathrm{~km} \mathrm{~s}^{-1}$ (Moffett et al. 1993; Katsuda et al. 2009), and $\eta \sim 1-10$ (Petruk et al. 2011), which means that for radio-emitting electrons the resulting acceleration time (less than a year) is extremely rapid compared to the SNR evolutionary timescale $(\sim 1 \mathrm{kyr})$. As a result, variations of radio morphology with obliquity point to electron injection physics alone, which make it the best regime in which to investigate this matter.

In this context, the remnants showing a radio shell with two opposite bright and regular limbs separated by two minima of emission (often called bilateral or barrel-shaped or bipolar, BSNRs hereafter, Kesteven \& Caswell 1987; Gaensler 1998) are considered ideal laboratories, because their morphology is definitely not heavily affected by small-scale inhomogeneities that could render the interpretations rather difficult. A pointlike supernova explosion that occurs in a uniform magnetized medium with a constant value and direction of the interstellar magnetic field (hereafter ISMF) yields a symmetric radio BSNR. The bright limbs are located where the magnetic field is parallel or perpendicular to the shock normal, if the injection efficiency is quasi-parallel or quasi-perpendicular/isotropic ${ }^{1}$, respectively, and if the ISMF direction lies in the plane of sky, whereas different configurations occurs at different aspect angles (Fulbright \& Reynolds 1990; Orlando et al. 2007).

${ }^{1}$ Here, perpendicular and parallel refers to the angle between the shock normal and the pre-shock magnetic field (this angle is called the obliquity angle). In particular, the injection is called quasi-parallel (quasi-perpendicular) if its efficiency is maximum where the obliquity angle is $0^{\circ}\left(90^{\circ}\right.$, Fulbright \& Reynolds 1990). The injection is called isotropic if its efficiency does not depend on the obliquity angle (Ellison et al. 1995; Völk et al. 2003). 
However, in the real universe, many SNRs evolve in a nonuniform ISM (see e.g. Hnatyk \& Petruk 1999, for an analytical treatment of SNR in nonuniform medium) and BSNRs are therefore often asymmetric. Orlando et al. (2007, hereafter Paper I) have generalized the study of Fulbright \& Reynolds (1990) to these cases by considering the explosions occurring in a largescale gradient of either density or magnetic field, showing that most of the asymmetries observed in true BSNRs can be recovered with this model. To be specific, the radio morphology loses one axis of symmetry, and the limbs are not equally bright (if the gradient runs across the limbs) or they are not opposite and converge on the side in which either the density or the magnetic field is increasing (if the gradient runs between the limbs).

Clearly, the morphology of BSNRs is tightly coupled with the magnetized environments in which the shock expands. Most remarkable is the preference of BSNR symmetry axis ${ }^{2}$ to be oriented parallel to the galactic plane, as reported by Gaensler (1998). It seems therefore possible to study the BSNR morphology to derive the geometry of the surrounding magnetic field, and thereby to investigate the microphysics of the particle acceleration processes that occur at the shock front. In this respect, BSNRs may be considered as a probe for structure of Galactic MF on scales of $\sim 10 \mathrm{pc}$, which is lower than the expected large scale variation of the field predicted by global models (Prouza \& Šmída 2003; Kachelrieß et al. 2007; Sun et al. 2008).

The remnant of SN1006 seems to be the object in which this kind of study may be most fruitful. The uniform environment and the bright limbs visible in most of the electromagnetic spectrum make it a real case-study in the field of particle acceleration mechanisms in strong shocks. Indeed, Rothenflug et al. (2004) applied a simple and powerful geometrical argument to the XMM-Newton X-ray image of SN1006, based on the ratio between the central and the rim luminosity. This showed that the bright limbs are polar caps (instead of an equatorial belt) and that, therefore, the magnetic field is oriented across the limbs, in the NE-SW direction. This argument seems to break the dichotomy between the two competing scenarios of the dependence of the injection efficiency on the obliquity angle $\theta$ (the angle between the magnetic field direction and the shock normal), preferring a situation in which the injection is most efficient when the field is along the shock normal (quasi-parallel scenario) over the situation in which the field is perpendicular (quasi-perpendicular scenario, see Fulbright \& Reynolds 1990).

On one hand, these findings were expected because Völk et al. (2003) already pointed out that in SN1006 the injection should be maximum at parallel shocks. However, in the light of the uncertainties related to the details of the acceleration processes, several authors still considered the quasiperpendicular scenario a viable option: Fulbright \& Reynolds (1990) argued against the quasi-parallel scenario pointing out that quasi-parallel models often give rise to unobserved morphologies in the radio band (see also Orlando et al. 2007); Yamazaki et al. (2004) still considered both models to explain the observed width of selected filaments of SN1006 observed by Bamba et al. (2003), whereas Amano \& Hoshino (2007) developed a quasi-perpendicular model that agrees very well with the same data.

Recently, Petruk et al. (2009b) have further investigated this problem, and showed that in the framework of a simple model of SN1006 in terms of a point-like explosion occurring in a uniform density and uniform magnetic field medium, there is no way to reconcile the quasi-parallel scenario and the SN1006

\footnotetext{
2 The axis running parallel to the limbs in the plane of the sky.
}

morphology as observed in the radio band. The same conclusion was reached more recently by Schneiter et al. (2010). The contradiction in the interpretation of the radio morphology (suggesting an isotropic or quasi-perpendicular injection efficiency scenario) and X-ray morphology (suggesting a quasiparallel scenario), and the fact that SN1006 shows both the kinds of asymmetries discussed in Paper I (converging limbs and different surface brightness), has prompted us to investigate the effects of non-uniformity of the environment on the observed properties of this remnant, capitalizing on the work of Orlando et al. (2007) on asymmetric BSNRs.

In Sect. 2 we briefly describe the MHD model used to reproduce the remnant of SN1006, which includes a small gradient of the magnetic field. In Sect. 3 we introduce the methodology for the comparison between modeled and observed radio images of SN1006, while in Sect. 4 we discuss the results of the comparison, showing that the model with the gradient of $|\boldsymbol{B}|$ is not only able to reconcile for the first time the interpretation of radio and $\mathrm{X}$-ray morphology of the remnant, but it also provides stringent constraints on the overall geometry of the field in the vicinity of the remnant.

\section{The model}

We adopted the 3D MHD SNR model described in Orlando et al. (2007), to which we refer the reader for further details, and used the FLASH code (Fryxell et al. 2000) for the model implementation. Because there is accumulated evidence that the density around SN1006 is fairly constant, with the exception of the NW sector where an encounter with a dense cloud is occurring (Acero et al. 2007; Miceli et al. 2009), we argue that a model with a $|\boldsymbol{B}|$ gradient is more appropriate than one with a density gradient to describe the asymmetries of the limbs of SN1006. Given the pronounced slantness of the limbs, we conclude, following Paper I, that a prominent component of $\nabla|\boldsymbol{B}|$ is directed parallel to the limbs (and therefore perpendicular to the galactic plane). The remnant was modeled as a point-like explosion of 1.4 solar masses of ejecta with a kinetic energy of $E=1.3 \times 10^{51} \mathrm{erg}$ occurring in a uniform density medium with $n_{0}=0.05 \mathrm{~cm}^{-3}$ (Acero et al. 2007). The ISMF has a value of $30 \mu \mathrm{G}$ in the environment of the explosion site. Such a high value for the location of SN1006 in the Galaxy was chosen to take into account, as a first approximation, the effects of magnetic field amplification, achieving a post-shock $|\boldsymbol{B}|$ on the order of a few hundreds of $\mu \mathrm{G}$ in our simulations, in agreement with observations of some SN1006 filaments (e.g. Yamazaki et al. 2004). The direction of the MF is along the $X$ axis while its gradient is along the $Z(X)$ axis for quasi-parallel (quasi-perpendicular) injection efficiency (see Fig. 1 of Paper I for more details). $\nabla|\boldsymbol{B}|$ values used in our simulations are reported in Table 1. The simulations were stopped at $t=1000 \mathrm{yr}$, checking that the shock velocity and remnant radius are compatible with the observed values (4600 $\mathrm{km} \mathrm{s}^{-1}$ and $9.6 \mathrm{pc}$, respectively).

We synthesized the radio emission using the REMLIGHT code of Orlando et al. (2011), to which we refer the reader for more details. In particular, the synchrotron radiation from relativistic electrons distributed with a power law spectrum $N(E) \propto$ $K E^{-s}$ is computed according to Ginzburg \& Syrovatskii (1965) with the formula $i(v) \propto K B_{\perp}^{(\alpha+1)} v^{-\alpha}$, where $\alpha=(s-1) / 2$, and $B_{\perp}$ is the component of the magnetic field perpendicular to the line-of-sight (LoS). The coefficient $K \propto \epsilon V_{\text {sh }}^{-b}$ includes all the dependencies from the obliquity and the shock velocity $\left(V_{\mathrm{sh}}\right)$ as described by Orlando et al. (2007), and, in particular, the injection efficiency is $\epsilon \propto \sin ^{2} \Theta_{\mathrm{Bn} 2}$ and $\epsilon \propto \cos ^{2} \Theta_{\mathrm{Bn} 2}$ in the 
Table 1. MHD models of SN1006 used in this work.

\begin{tabular}{lc}
\hline \hline Name & $\nabla|\boldsymbol{B}|^{a}$ \\
\hline UNIFORM_B & 1.0 \\
GRAD1 & 1.2 \\
GRAD2 & 1.4 \\
GRAD3 & 2.0 \\
GRAD4 & 4.7 \\
\hline
\end{tabular}

Notes. ${ }^{(a)}$ The relative variation of the magnetic field strength over a scale of $10 \mathrm{pc}$.

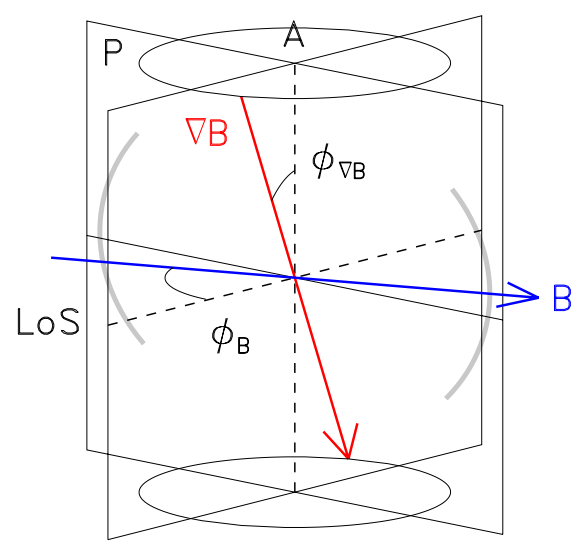

Fig. 1. Angles defining the viewing geometry, namely $\phi_{\boldsymbol{B}}$ (the magnetic field aspect angle) and $\phi_{\nabla|B|}$ (the rotation angle of the gradient). $P$ is the plane of the sky, on which the SN1006 are projected (sketched as light grey). See text for more details.

quasi-perpendicular and quasi-parallel case, where $\Theta_{\mathrm{Bn} 2}$ is the obliquity angle between the shock normal and the post-shock magnetic field. This particular choice of the obliquity dependance derives from the work of Fulbright \& Reynolds (1990), and it is largely adopted in the literature (e.g. Orlando et al. 2007; Schneiter et al. 2010; Orlando et al. 2011; for alternative formulations see Völk et al. 2003; and Petruk et al. 2009a). The parameter $b$ was chosen to be equal to -1.5 , reflecting a situation in which stronger shocks inject particle more efficiently, but we verified that our results are unaffected by choices of $b$ in the range $(-1.5,+2.0)$, the range explored by Orlando et al. $(2007)$.

\section{Comparison between models and observations}

\subsection{The angles defining the viewing geometry}

Several works in the literature show that the observed morphology of an SNR emitting synchrotron emission is strongly affected by the angle between the direction of $\boldsymbol{B}$ and the line of sight, $\phi_{\boldsymbol{B}}$. Moreover, because we also have a gradient of the magnetic field in our model, we are forced to consider the dependence of the observed morphology from the angle between the direction of the gradient and the plane of the sky, $\phi_{\nabla|B|}$. The definitions of $\phi_{\boldsymbol{B}}$ and $\phi_{\nabla|\boldsymbol{B}|}$ are sketched in Fig. 1. For the purpose of this work, we chose to synthesize our maps in the following way: the starting configuration has a MF perpendicular to the $\operatorname{LoS}\left(\phi_{\boldsymbol{B}}=90^{\circ}\right)$, and $\nabla|\boldsymbol{B}|$ lying in the plane of the sky (the plane $P$ in Fig. 1) and parallel to the lobes. First, we applied a rotation of $\phi_{\nabla|B|}$ degrees around the axis in the plane of the sky passing through the center of the remnant and perpendicular to the limbs, where positive angles mean that the region of increasing $\boldsymbol{B}$ is closer to us. Then, we applied a rotation of $90^{\circ}-\phi_{\boldsymbol{B}}$ degrees around the axis in the plane of the sky passing through the remnant center and parallel to the limbs (axis $A$ in Fig. 1). Others rotation schemes give similar results. The adopted values of $\phi_{\boldsymbol{B}}$ are from $0^{\circ}$ to $90^{\circ}$ in steps of $2^{\circ}$ and the ones of $\phi_{\nabla|\boldsymbol{B}|}$ are from $0^{\circ}$ to $90^{\circ}$ in steps of $15^{\circ}$. Therefore, we generated 315 maps for each model that encompass all combinations of the relevant angles.

\subsection{The morphological parameters}

The exploration of the parameter space is a challenging task because it involves the morphological comparison between many model maps and the true SN1006 image. We devised a simple procedure that involves the comparison of the value of four morphological parameters calculated both from the synthesized radio emission maps and from the observed radio map of SN1006. The parameters are the ratio between the maximum and the minimum of surface brightness $\left(S_{\mathrm{b}}\right)$ around the rim $(A)$, the ratio between the maximum around the rim and the center of the remnant $(C)$, the ratio of $S_{\mathrm{b}}$ of the two bright limbs $\left(R_{\max }\right)$, and the angular separation between the maxima of the limbs $\left(\theta_{\mathrm{D}}\right)$. The parameters $A, R_{\max }$ and $\theta_{\mathrm{D}}$ have also been used in Orlando et al. (2007), and we refer to that paper for further discussion. The $C$ parameter is introduced here to measure the luminosity contrast between the brightest rim and the center. Note that the $C$ parameter does not correspond to the $R_{\pi / 3}$ parameter that was used by Rothenflug et al. (2004) to exclude the equatorial belt scenario for SN1006, because $C$ is measured in a small circular region in the radio map (see below). While $R_{\max }$ and $\theta_{\mathrm{D}}$ gives a measure of the asymmetries (if any) in the remnant morphology, $A$ and $C$ give the idea of the remnant being centrally filled $(C>1, A \sim 1)$ or barrel-shaped $(C<1, A>1)$. This choice of evaluating the emission in selected regions makes the method less sensitive to the exact functional form of the obliquity dependance of the injection efficiency introduced in Sect. 2.

The parameters $A, C$, and $R_{\max }$ are measured for SN1006 using the radio map of Petruk et al. (2009b), by averaging the $S_{\mathrm{b}}$ value on circular regions of $45^{\prime \prime}$ radius (Fig. 2). The values we derived are $A=2.7 \pm 0.2, C=0.36 \pm 0.03, R_{\max }=1.2 \pm 0.1$, and $\theta_{\mathrm{D}}=135^{\circ} \pm 10^{\circ}$. For the measurement of the parameters in the synthesized radio maps we used an automated procedure to find the maximum of the two limbs, the minimum between limbs along the rim and the central position. Then we used an average in circular regions whose radius is the same percentage as the remnant radius used in the true radio image $(5 \%)$ to minimize fluctuations. Because the model images were synthesized using the same number of pixels per remnant radius as the true radio map, this procedure ensures that the model and observed values of the parameters are comparable.

\subsection{Test of the procedure}

To test the procedure, we ran the model with the initial parameters discussed in Sect. 2, but assuming a uniform magnetic field in the quasi-parallel, quasi-perpendicular and isotropic injection efficiency scenarios. This situation already contradicts the observed values $\theta_{\mathrm{D}}=135^{\circ}$ and $R_{\max }=1.2$, because $\nabla|\boldsymbol{B}|=0$ implies $\theta_{\mathrm{D}}=180^{\circ}$ and $R_{\max }=1$. However, this MF configuration has been used in the literature, and it is a good test case for our procedure. In Fig. 3 we show the $A-C$ scatter plot for various $\phi_{\boldsymbol{B}}$ angles in the uniform $\boldsymbol{B}$ case and quasi-parallel, 


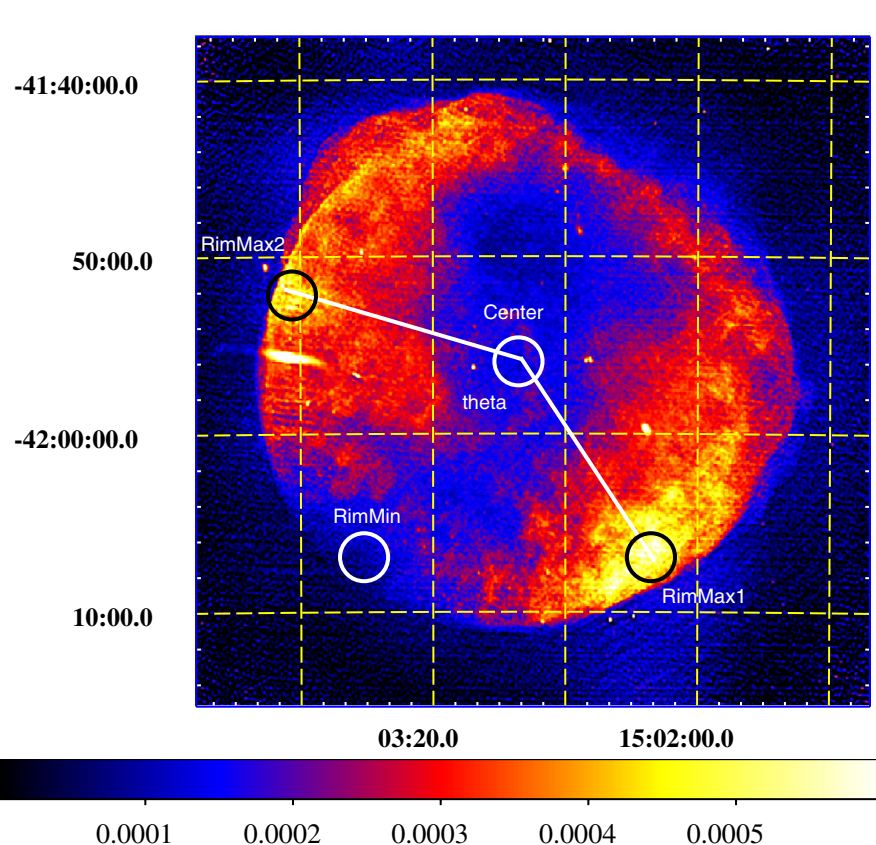

Fig. 2. Radio map of SN1006 at $1.4 \mathrm{GHz}$ with the four regions in which the surface brightness of the remnant was averaged for the purpose of computation of the four morphological parameters $A=$ $\mathrm{RIM}_{\max } / \mathrm{RIM}_{\min }, C=$ center $/ \mathrm{RIM}_{\max }, R_{\max }=\mathrm{RIM}_{\max 1} / \mathrm{RIM}_{\max 2}$ and $\theta_{\mathrm{D}}$, the aperture angle between the limb maxima. Adapted from Petruk et al. (2009b).

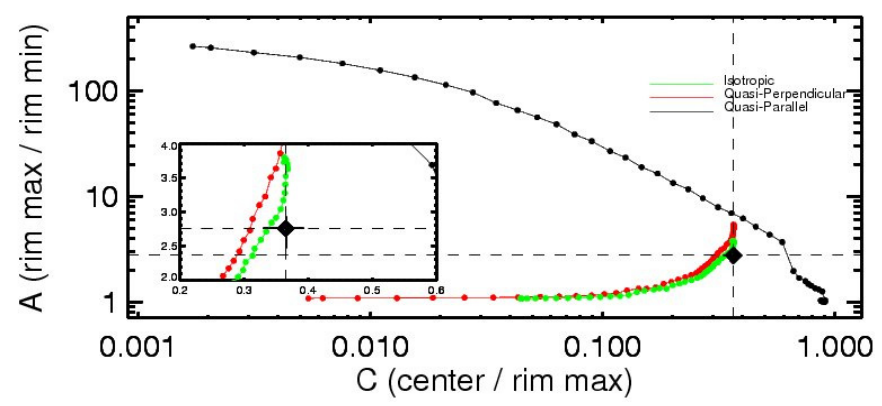

Fig. 3. Comparison between synthesized radio maps for a uniform $\boldsymbol{B}$ at an age of $1000 \mathrm{yr}$ (black, green, and red dots for quasi-parallel, quasiperpendicular, and isotropic injection efficiency, respectively) and the observed radio map (black diamond, the inset shows a zoom of the plot around this point) by means of the use of the $\mathrm{A}$ and $\mathrm{C}$ morphological parameters introduced in Fig. 2. Each curve represents the values spanned by the parameters when the aspect angle $\phi_{\boldsymbol{B}}$ goes from $0^{\circ}$ (rightmost part) to $90^{\circ}$ (leftmost part).

quasi-perpendicular and isotropic scenarios ${ }^{3}$. In this case, the plot suggests that the quasi-parallel model does not reproduce the observed parameters at all, unlike the quasi-perpendicular and isotropic scenarios, whose best-fit is $\phi_{\boldsymbol{B}} \approx 70^{\circ}$. This excellently agrees with the work of Petruk et al. (2009b), who derived $\phi_{\boldsymbol{B}}$ by comparing the azimuthal brightness profile with models in a uniform magnetic field. Therefore, we conclude that our method is robust.

\footnotetext{
$\overline{{ }^{3} \text { In the uniform } \boldsymbol{B} \text { case, } R_{\max }}=1$ and $\theta_{\mathrm{D}}=180^{\circ}$ by definition.
}

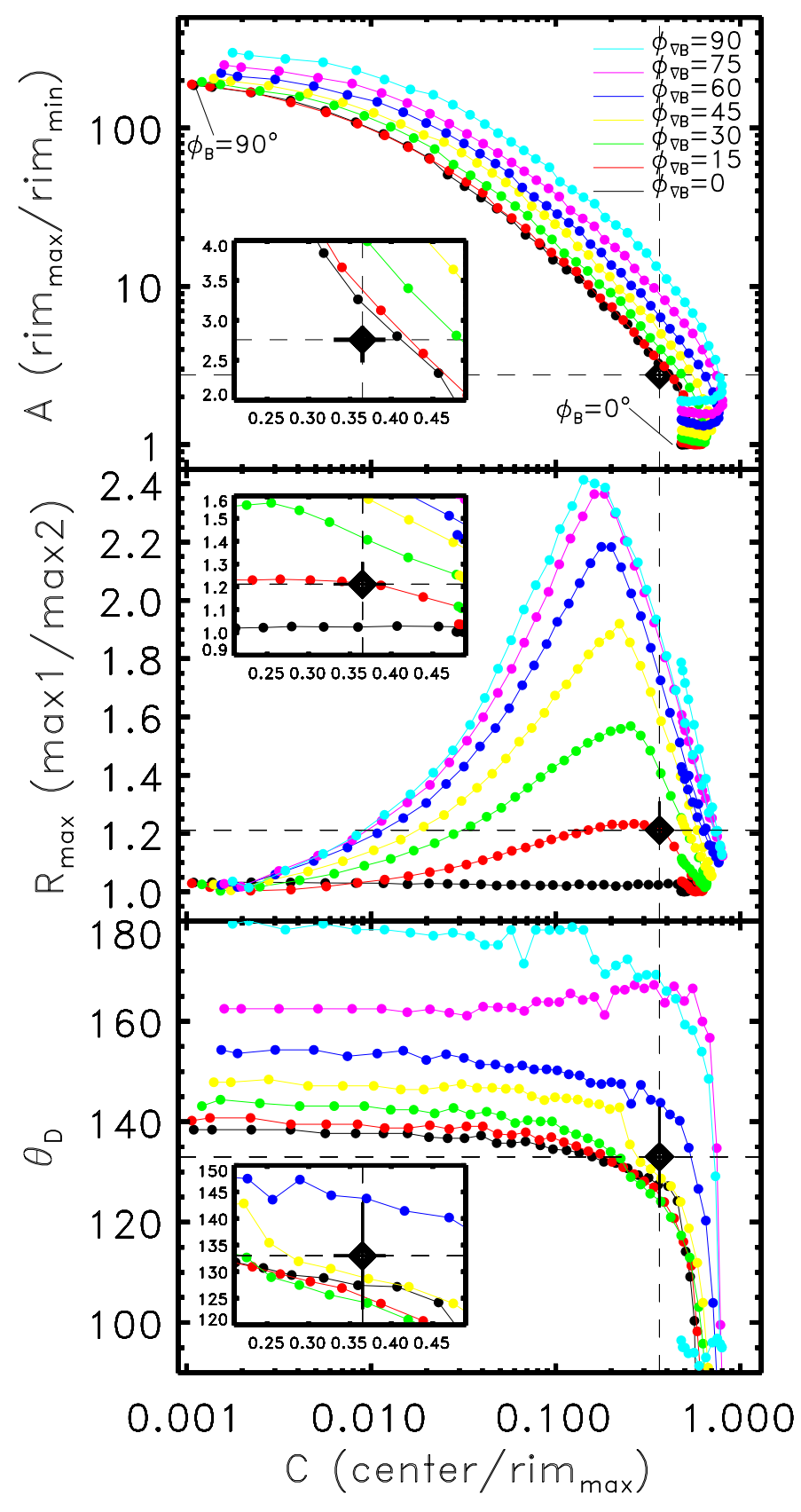

Fig. 4. Top panel: $A-C$ scatter plot for our model GRAD2 (Table 1), assuming quasi-parallel injection and an age of $1000 \mathrm{yr}$. Different colors correspond to different values of $\phi_{\nabla|B|}\left(\right.$ from $0^{\circ}$ to $90^{\circ}$ in steps of $15^{\circ}$ ), whereas each dot corresponds to a given value of $\phi_{\boldsymbol{B}}$ (from $0^{\circ}$, right, to $90^{\circ}$, left, in steps of $\left.2^{\circ}\right)$. We overplotted the $(A, C)$ values measured for SN1006 (diamonds; the inset shows a zoom near this point). Middle panel: same as top panel but for the $R_{\max }-C$ pair. Lower panel: same as top panel but for the $\theta_{\mathrm{D}}-C$ pair.

\subsection{Results}

\subsubsection{Polar caps}

In Fig. 4 we show the $A-C, R_{\max }-C$ and $\theta_{\mathrm{D}}-C$ scatter plots computed in the synthesized radio maps of our SN1006 model including a weak gradient of the magnetic field (GRAD2 in Table 1, i.e., $\boldsymbol{B}$ varies by a factor 1.4 over 10 pc scale), and assuming that the electron injection efficiency is maximum where the obliquity angle is $0^{\circ}$ (quasi-parallel scenario). This 

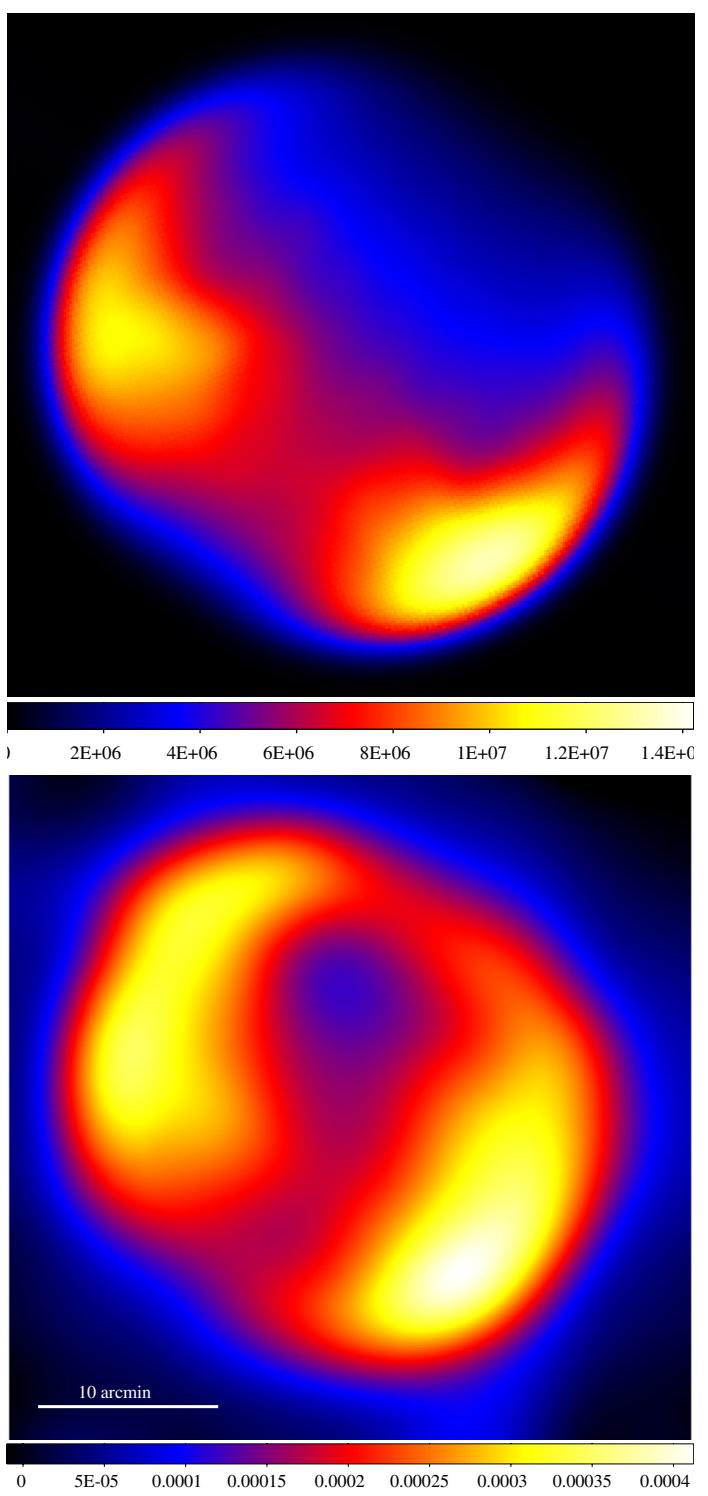

Fig. 5. Top panel: synthesized radio image at $1 \mathrm{GHz}$ for our best-fit model (GRAD2 in Table 1, and quasi-parallel injection efficiency scenario, $\phi_{B}=38^{\circ}$ and $\phi_{\nabla|B|}=15^{\circ}$ ), smoothed with a sigma of $2^{\prime}$. Bottom panel: radio map of SN1006 of Fig. 2, but smoothed with the same sigma as for the model image.

configuration has also been referred to as "polar caps". We also overplotted the values of the parameters derived using the true radio map of the remnant of Petruk et al. (2009b). Though the agreement is not exactly perfect in all the plots, we note that we can define a very limited region of the parameter space $\left(\phi_{\boldsymbol{B}}, \phi_{\nabla|\boldsymbol{B}|}\right)$ which is compatible with the observed values of $A$, $C, R_{\max }$ and $\theta_{\mathrm{D}}$. This means that the observed radio morphology of SN1006 is overall compatible with a quasi-parallel scenario for this remnant, if we include a weak gradient of $\boldsymbol{B}$.

A remarkable result is that the comparison between the quasi-parallel model and the observation strongly excludes a situation in which the polar caps are in the plane of the sky $\left(\phi_{\boldsymbol{B}}=90^{\circ}\right)$ or along the line of sight $\left(\phi_{\boldsymbol{B}}=0^{\circ}\right)$. The latter geometry would cause a centrally brightened radio morphology instead of two limbs, as already discussed by Fulbright \& Reynolds (1990) and Orlando et al. (2007). The best-fit values of the relevant angles derived from Fig. 4 and a conservative estimate of their uncertainties are $\phi_{\boldsymbol{B}}=38^{\circ} \pm 4^{\circ}$ and $\phi_{\nabla|\boldsymbol{B}|}=15^{\circ} \pm 15^{\circ}$.
The synthesized radio map of the best-fit model is shown in Fig. 5 (top panel) along with the observed radio map (bottom panel). Both maps were slightly smoothed ( $2^{\prime}$ sigma, $\sim 1 / 15$ the remnant size) to focus the comparison on the general obliquity trends. Indeed, we are not interested in reproducing the fine structure of the thermal emission from the ejecta that is visible in the high-resolution radio observation. We note that the largescale structures of the observed radio emission are very well recovered by the best-fit model and the two images look similar.

The comparison with other gradients, namely the GRAD1 (weaker gradient), GRAD3 and GRAD4 models (stronger gradient), shows that it is impossible to find a satisfactory fit for all parameters. In particular, for higher values of the gradient, the angle $\theta_{\mathrm{D}}$ is always smaller than observed in most of the cases, and for lower values it is always larger, so GRAD2 is the only model which gives us an overall good fit. The variation of the MF in SN1006 can therefore be bracketed between 1.2 and 2.0 (see Table 1).

\subsubsection{Equatorial belt}

In this section, we explore the same MHD model as in the previous section, but now assuming that the electron injection efficiency is maximum where the obliquity angle is $90^{\circ}$ (quasiperpendicular scenario). This configuration has also been refereed to as "equatorial belt". We produced the synthetic images and repeated the analysis described in the previous paragraphs. In this case, the direction of the $\nabla|\boldsymbol{B}|$ is aligned with the direction of $\boldsymbol{B}^{4}$, so the angle $\phi_{\nabla|\boldsymbol{B}|}$ is the same as $\phi_{\boldsymbol{B}}$, and we did not consider it any further. Another consequence of this is that $R_{\max }$ is always 1 (in disagreement with observations), so this parameter cannot give any diagnostics. The results are shown in Fig. 6, and it seems that a good fit can be found for $\phi_{\boldsymbol{B}} \sim 70^{\circ}$ (a value that agrees with the azimuthal profile analysis of Petruk et al. 2009b), even if the model points in the $\theta_{\mathrm{D}}-C$ diagram seem to be more distant from the observed points than any scatter plot in Fig. 4, whch indicates a better fit in the quasi-parallel case then in the quasi-perpendicular one.

\section{Discussion}

We introduced a new method of comparison between models and observations of radio SNRs shells that can provide useful information on the 3D structure of the remnant and on the unperturbed ambient magnetic field in which the remnant expands. The method is based on the calculation of four morphological parameters. For SN1006, we found that the method gives a good fit if the injection efficiency is quasi parallel and the ambient magnetic field is characterized by a gradient of its strength. In addition, the method allowed us to constrain the viewing geometry angles of the magnetic field $\left(\phi_{\boldsymbol{B}}=38^{\circ} \pm 4^{\circ}\right.$ and $\phi_{\nabla|B|}=15^{\circ} \pm 15^{\circ}$ ). Schneiter et al. (2010) showed that the quasiperpendicular model is a better fit to the $R_{\pi / 3}$ value (the ratio between the inner region and the limb region flux introduced by Rothenflug et al. 2004) of SN1006 in the radio band, but they did not properly consider aspect angles $\neq 90^{\circ}$ and the non-uniform magnetic field. We verified that our best-fit quasi-perpendicular model gives $R_{\pi / 3}=0.16$ for $\phi_{B}=90^{\circ}$, but $R_{\pi / 3}=1.00$ for

\footnotetext{
${ }^{4}$ This is strictly necessary because Orlando et al. (2007) has shown that the gradient increasing direction is parallel to the limb, so quasiperpendicular injection efficiency implies $\boldsymbol{B}$ perpendicular to shock normal at the limbs and therefore $\boldsymbol{B}$ parallel to $\nabla|\boldsymbol{B}|$.
} 


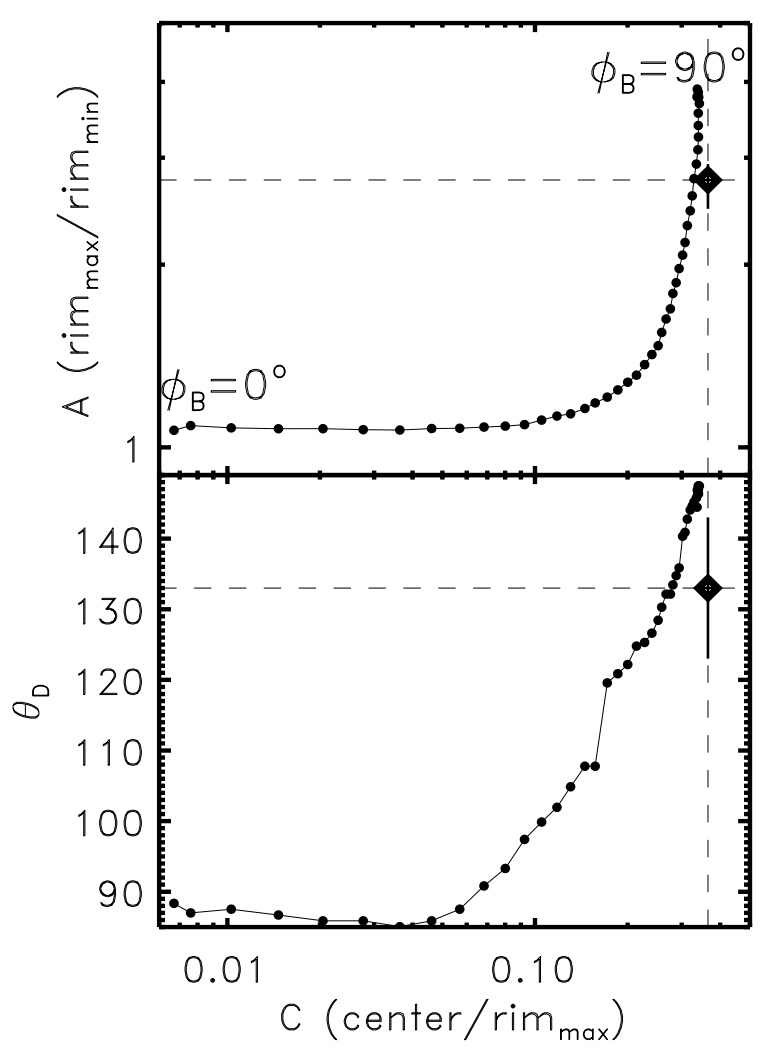

Fig. 6. Top panel: same as Fig. 4 (top panel) but for the GRAD2 model and quasi-perpendicular scenario. There is no $\phi_{\nabla|B|}$ angle shown in this case, because $\boldsymbol{B}$ is always aligned with $\nabla|\boldsymbol{B}|$. Lower panel: scatter plot of $\theta_{\mathrm{D}}-C$ parameters. We overplotted the values observed in SN1006 (diamonds).

$\phi_{\boldsymbol{B}}=38^{\circ}$, in agreement with the value measured in the new radio map of Fig. $2\left(R_{\pi / 3}^{\text {obs }}=1.01\right)$.

The symmetry axis of SN1006 is perpendicular to the galactic plane ${ }^{5}$, its distance is $2.2 \mathrm{kpc}$ (Winkler et al. 2003) and its galactic latitude is $14 \cdot 6^{\circ}$. Combining all these pieces of information, we can plot the direction of the magnetic field and its gradient in a 3D representation of the Galactic disk. This is shown in Fig. 7.

Quite remarkably, our best-fit values of $\phi_{\nabla|\boldsymbol{B}|}$ and $\phi_{\boldsymbol{B}}$ imply that the direction of $\nabla|\boldsymbol{B}|$ resulting from the fit to the quasiparallel model points down toward the Galactic plane and the direction of $\boldsymbol{B}$ is aligned with the direction of the spiral arm near the remnant. This is indeed a very reasonable configuration for the magnetic field of the Galaxy, so it is tempting to conclude that we are sampling the large-scale field of the Galaxy. However, we note that the best-fit value of $\nabla|\boldsymbol{B}|$ (i.e., a variation by a factor 1.4 over $10 \mathrm{pc}$, model GRAD2 in Table 1) seems to be high compared with the values predicted by current models of galactic MF. We used the model of Sun et al. (2008) to compute the expected variation of the azimuthal field in the Galaxy at the location of SN1006. In particular, we used their ASS+RING model, which seems to be one favored by rotation measures of pulsars in the plane. At the SN1006 distance of $\sim 550 \mathrm{pc}$ from the plane, the large-scale field is expected to vary by a factor of 1.4 on scales of more then $100 \mathrm{pc}$, much larger than the scale-length required by our lowest gradient model (GRAD1 in Table 1). This

\footnotetext{
5 This makes SN1006 rather peculiar, because the majority of bilateral SNRs have their symmetry axis parallel to the galactic plane (Gaensler 1998).
}

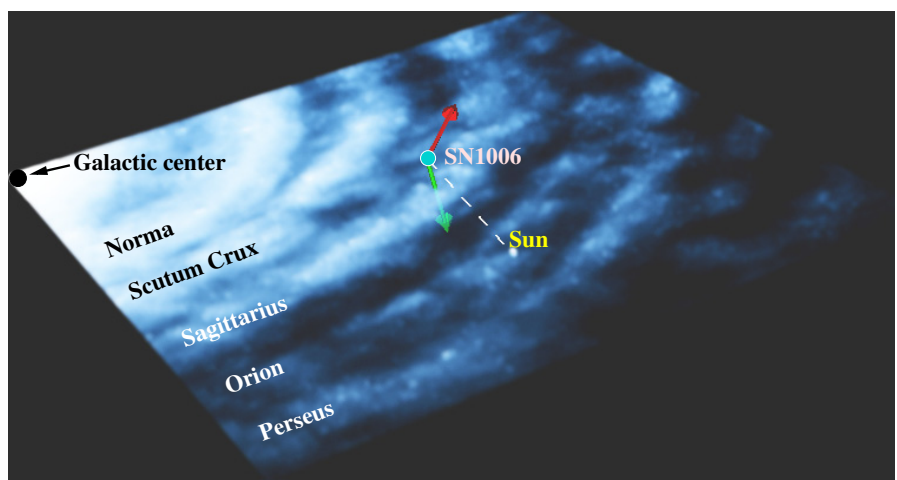

Fig. 7. Artist view of the magnetic field at the location of SN1006 in our Galaxy. The red and green arrows mark the directions of the field and its gradient as derived by the best-fit model for the synchrotron radio emission of SN1006, assuming a quasi-parallel scenario as derived in this work. The figure is in scale.

opens up the possibility that we are actually sampling the random magnetic field component. Minter \& Spangler (1996) report length scales for this component on the order of few pc, which is compatible with the variations we derive.

\section{Summary and conclusion}

The synchrotron radio emission of the archetypical bilateral supernova remnant SN1006 is compared with a MHD model for this remnant, including a gradient of the magnetic field strength, particle acceleration and its obliquity dependent characteristic, namely quasi-perpendicular, quasi-parallel and isotropic scenarios for the injection efficiency and a rough treatment of magnetic field amplification. To explore the parameters space, which is very large owing to the necessity to include the viewing geometry in the model-data comparison, we developed a simplified procedure based on the computation of four morphological parameters. We found a very good fit with a model assuming quasi-parallel injection efficiency and a configuration of the ambient magnetic field characterized by a variation of its strength of abut a factor of $1.4(1.2-2.0)$ over a scale of $10 \mathrm{pc}$, and by viewing geometry angles $\phi_{\boldsymbol{B}}=38^{\circ} \pm 4^{\circ}$ (between the direction of $\boldsymbol{B}$ and the line of sight), and $\phi_{\nabla|B|}=15^{\circ} \pm 15^{\circ}$ (between the plane of the sky and the direction of the gradient of the magnetic field). A worse fit is obtained in the quasi-perpendicular scenario. The overall morphology of the observed radio emission at $1.4 \mathrm{GHz}$ is correctly recovered by our best-fit model. Therefore, we conclude that the SN1006 limbs are polar caps, significantly tilted with respect to the plane of the sky. The projected direction of $\boldsymbol{B}$ and $\nabla|\boldsymbol{B}|$ in the Galaxy are along the spiral arm and toward the plane respectively, which very well agrees with the expected direction of the large-scale galactic $\boldsymbol{B}$. However, the implied gradient is too high to be associated to the large-scale galactic $\boldsymbol{B}$ at the location of the SN1006 remnant and more typical of the random magnetic field components.

The application of our method to selected samples of bilateral supernova remnants may yield independent estimates of the geometry of the galactic field at several locations, which can be useful for understanding the field topology in our Galaxy.

Acknowledgements. F.B. acknowledges many fruitful discussions and the friendly environment at the ISSI meeting on "Magnetic field in the universe", and in particular with Aris Noutsos. The software used in this work was in part developed by the DOE-supported ASC/Alliances Center for Astrophysical Thermonuclear Flashes at the University of Chicago. The calculations were 
performed on the cluster at the SCAN (Sistema di Calcolo per l'Astrofisica Numerica) facility of the INAF Osservatorio Astronomico di Palermo.

\section{References}

Acero, F., Ballet, J., \& Decourchelle, A. 2007, A\&A, 475, 883

Amano, T., \& Hoshino, M. 2007, ApJ, 661, 190

Ballet, J. 2006, Adv. Space Res., 37, 1902

Bamba, A., Yamazaki, R., Ueno, M., \& Koyama, K. 2003, ApJ, 589, 827

Blasi, P., Gabici, S., \& Vannoni, G. 2005, MNRAS, 361, 907

Ellison, D. C., Baring, M. G., \& Jones, F. C. 1995, ApJ, 453, 873

Fryxell, B., Olson, K., Ricker, P., et al. 2000, ApJS, 131, 273

Fulbright, M. S., \& Reynolds, S. P. 1990, ApJ, 357, 591

Gaensler, B. M. 1998, ApJ, 493, 781

Ginzburg, V. L., \& Syrovatskii, S. I. 1965, ARA\&A, 3, 297

Hnatyk, B., \& Petruk, O. 1999, A\&A, 344, 295

Kachelrieß, M., Serpico, P. D., \& Teshima, M. 2007, Astropart. Phys., 26, 378

Katsuda, S., Petre, R., Long, K. S., et al. 2009, ApJ, 692, L105

Kesteven, M. J., \& Caswell, J. L. 1987, A\&A, 183, 118

Miceli, M., Bocchino, F., Iakubovskyi, D., et al. 2009, A\&A, 501, 239

Minter, A. H., \& Spangler, S. R. 1996, ApJ, 458, 194
Moffett, D. A., Goss, W. M., \& Reynolds, S. P. 1993, AJ, 106, 1566

Morlino, G., Amato, E., Blasi, P., \& Caprioli, D. 2010, MNRAS, 405, L21

Orlando, S., Bocchino, F., Reale, F., Peres, G., \& Petruk, O. 2007, A\&A, 470, 927

Orlando, S., Petruk, O., Bocchino, F., \& Miceli, M. 2011, A\&A, 526, A129

Petruk, O., Beshley, V., Bocchino, F., \& Orlando, S. 2009a, MNRAS, 395, 1467

Petruk, O., Dubner, G., Castelletti, G., et al. 2009b, MNRAS, 393, 1034

Petruk, O., Beshley, V., Bocchino, F., Miceli, M., \& Orlando, S. 2011, MNRAS, 413, 1643

Prouza, M., \& Šmída, R. 2003, A\&A, 410, 1

Rothenflug, R., Ballet, J., Dubner, G., et al. 2004, A\&A, 425, 121

Schneiter, E. M., Velázquez, P. F., Reynoso, E. M., \& de Colle, F. 2010, MNRAS, 408, 430

Sun, X. H., Reich, W., Waelkens, A., \& Enßlin, T. A. 2008, A\&A, 477, 573

Uchiyama, Y., Aharonian, F. A., Tanaka, T., Takahashi, T., \& Maeda, Y. 2007, Nature, 449, 576

Völk, H. J., Berezhko, E. G., \& Ksenofontov, L. T. 2003, A\&A, 409, 563

Völk, H. J., Berezhko, E. G., \& Ksenofontov, L. T. 2005, A\&A, 433, 229

Winkler, P. F., Gupta, G., \& Long, K. S. 2003, ApJ, 585, 324

Yamazaki, R., Yoshida, T., Terasawa, T., Bamba, A., \& Koyama, K. 2004, A\&A 416,595 